\title{
Stochastic Models for the Kinematics of Moisture Transport and Condensation in Homogeneous Turbulent Flows
}

\author{
Paul A. O'Gorman and TAPio Schneider \\ California Institute of Technology, Pasadena, California
}

(Manuscript received 17 November 2005, in final form 3 March 2006)

\begin{abstract}
The transport of a condensing passive scalar is studied as a prototype model for the kinematics of moisture transport on isentropic surfaces. Condensation occurs whenever the scalar concentration exceeds a specified local saturation value. Since condensation rates are strongly nonlinear functions of moisture content, the mean moisture flux is generally not diffusive. To relate the mean moisture content, mean condensation rate, and mean moisture flux to statistics of the advecting velocity field, a one-dimensional stochastic model is developed in which the Lagrangian velocities of air parcels are independent OrnsteinUhlenbeck (Gaussian colored noise) processes. The mean moisture evolution equation for the stochastic model is derived in the Brownian and ballistic limits of small and large Lagrangian velocity correlation time. The evolution equation involves expressions for the mean moisture flux and mean condensation rate that are nonlocal but remarkably simple. In a series of simulations of homogeneous two-dimensional turbulence, the dependence of mean moisture flux and mean condensation rate on mean saturation deficit is shown to be reproducible by the one-dimensional stochastic model, provided eddy length and time scales are taken as given. For nonzero Lagrangian velocity correlation times, condensation reduces the mean moisture flux for a given mean moisture gradient compared with the mean flux of a noncondensing scalar.
\end{abstract}

\section{Introduction}

Studies of the Lagrangian moisture transport suggest that, sufficiently far away from regions of moist convection, large-scale processes significantly influence the relative humidity of the free troposphere. (e.g., Yang and Pierrehumbert 1994; Sherwood 1996; Salathé and Hartmann 1997; Pierrehumbert and Roca 1998; Dessler and Sherwood 2000). Galewsky et al. (2005) have shown that large-scale aspects of the relative humidity distribution in the free troposphere can be reproduced by averaging over Lagrangian trajectories of air parcels if one assumes that the specific humidity of air parcels is the specific humidity at last large-scale saturation. For example, subtropical air in the free troposphere is a mixture of relatively moist air that was last saturated in the deep Tropics or in the boundary layer and relatively dry air that was last saturated at higher levels in the atmosphere. A significant fraction of air parcels in

Corresponding author address: Paul O'Gorman, California Institute of Technology, Mail Code 100-23, 1200 E. California Blvd., Pasadena, CA 91125.

E-mail: pog@caltech.edu the driest regions of the subtropics, according to Galewsky et al., has last been saturated at points lying poleward and upward approximately on the isentrope going through the dry region of the subtropics. This indicates that, as suggested by Kelly et al. (1991) and Yang and Pierrehumbert (1994), subtropical air parcels are carried poleward and upward by large-scale eddies, cool adiabatically with attendant condensation and drying along their trajectories, and return to the subtropics drier than they were originally. At the same time, largescale eddy transport out of the deep Tropics provides a counterbalancing moisture source for the subtropics [Pierrehumbert (1998); see Schneider et al. (2006) for observational support for this general picture].

To account for the maintenance and variability of the tropospheric relative humidity and to account for results such as that the relative humidity stays approximately constant as the climate changes moderately (Held and Soden 2000; Soden et al. 2005), it is therefore necessary to relate relative or specific humidities, moisture fluxes, and condensation rates to large-scale eddy statistics. Here we develop stochastic models that provide such relations. We take eddy length and time scales as given and study how the mean humidity, mean 
condensation rate, and mean moisture flux depend on them. We treat moisture as a passive scalar that condenses whenever its concentration exceeds a specified local saturation value, and we assume parcel trajectories to be confined to two-dimensional surfaces, taken to represent dry or moist isentropes. We ignore the asymmetry of parcel trajectories due to the fact that adiabatically ascending saturated parcels approximately follow saturated moist isentropes, whereas adiabatically descending parcels approximately follow dry isentropes (Shaw 1930, chapter 6; Yang and Pierrehumbert 1994). We also ignore feedbacks of the moisture field on eddy dynamics, such as through latent heat release or through the radiative effects of water vapor. That is, we postpone dynamical questions and focus on the kinematics of moisture transport and condensation. While this considerably simplifies the problem, moisture statistics are nonetheless difficult to calculate because condensation introduces a strong nonlinearity into the specific humidity evolution equation.

Various approaches have been proposed to parameterizing the mean moisture flux and mean condensation rate- the principal unclosed terms in the evolution equation for mean specific humidity. Stone and Yao (1990) developed a two-dimensional zonally averaged general circulation model with a hydrological cycle, parameterizing the mean meridional eddy moisture flux by making a constant relative humidity assumption for moisture fluctuations, linearizing the ClausiusClapeyron relation, and relating the eddy moisture flux to the eddy heat flux. They parameterized large-scale condensation as a process that prevented supersaturation in the zonal mean, rather than locally. Watterson (1998) and Vallis (1982) used mixing-length approaches to parameterize the moisture flux, with Vallis (1982) parameterizing large-scale condensation by relaxing the zonal-mean relative humidity to a critical value whenever the critical value is exceeded. These approaches assume that the relative humidity is given, rather than internally determined; or that the mean moisture flux is diffusive, which is a questionable assumption, given the nonlinearity introduced in the specific humidity evolution equation by condensation.

Our approach builds on that of Pierrehumbert et al. (2006), who studied a stochastic model with independent stochastic processes for the Lagrangian velocities of air parcels, each carrying a value of specific humidity that is reset to a specified local saturation value whenever the saturation value is exceeded. Pierrehumbert et al. (2006) considered initial value and equilibrium problems using a white noise process for the velocity and with the saturation specific humidity fixed in time. Their model captures fundamental nonlinear effects of condensation; see also Sukhatme and Pierrehumbert (2006, manuscript submitted to Phys. Rev. E) for a related study of the moisture probability density function. We consider stochastic processes for the velocity with nonzero correlation time because of their relevance for atmospheric flows. We focus on the evolution equation for mean specific humidity and derive exact expressions for the mean moisture flux and mean condensation in the limits of small or large velocity correlation time. With a series of simulations of homogeneous twodimensional turbulence advecting a condensible scalar with an imposed mean gradient, we show that the onedimensional stochastic model can account for the kinematics of moisture transport and condensation in turbulent flows.

Section 2 describes an idealized advection-condensation system representing moisture transport and condensation on a two-dimensional surface. Section 3 discusses the one-dimensional stochastic advectioncondensation model and the evolution equation for mean specific humidity. Section 4 discusses simulations of homogeneous two-dimensional turbulence advecting a condensible moisture tracer and examines the extent to which results from the stochastic model can account for moisture kinematics in the simulations. Section 5 summarizes the conclusions.

\section{Idealized advection-condensation system}

We consider a two-dimensional advectioncondensation system as an idealized representation of eddy moisture transport along isentropes in the free troposphere. Our aim is to study how condensation affects the mean moisture flux and mean condensation. The moisture tracer is taken to be passive and so does not affect the advecting flow through latent heat release or radiative effects. Following Pierrehumbert et al. (2006), we make the simplifying assumption that the saturation specific humidity $q_{s}$ decreases monotonically toward the poles but has no zonal or time fluctuations. This allows us to capture the mean decrease in $q_{s}$ poleward along isentropes due to adiabatic cooling but not fluctuations in $q_{s}$ due to temperature fluctuations in eddies.

The equation for the specific humidity $q(\mathbf{x}, t)$ is

$$
\frac{\partial q}{\partial t}+\mathbf{u} \cdot \nabla q=-c,
$$

where $\mathbf{x}=(x, y)$ is the position, with zonal coordinate $x$ and meridional coordinate $y$, and $\mathbf{u}=(u, v)$ is the two-dimensional advecting velocity. The condensation rate $c(\mathbf{x}, t)$ is nonnegative and only nonzero when $q(\mathbf{x}$, $t)=q_{s}(y)$. It instantaneously ensures that supersatura- 
tion, $q \geq q_{s}$, does not occur, and may be defined formally as

$$
c(\mathbf{x}, t)= \begin{cases}-\mathbf{u} \cdot \nabla q & \text { if } q=q_{s} \text { and } \mathbf{u} \cdot \nabla q<0 \\ 0 & \text { otherwise. }\end{cases}
$$

The effect of condensation on moisture transport is of interest not only for the large-scale quasi-horizontal transport discussed here but also for vertical transport and smaller-scale turbulent flows. The moisture evolution Eq. (1) is the simplest equation that can capture the interplay between advection and condensation. Despite the simplifications that have been made, the nonlinearity of the condensation term renders the problem of obtaining a closed evolution equation for the mean specific humidity more difficult than the classical turbulence closure problem for a noncondensing passive scalar. We discuss the behavior of the condensible passive scalar in turbulent flows, taking the statistics of the much studied noncondensing passive scalar as given.

\section{One-dimensional stochastic model}

Assuming that the meridional velocity $v$ is independent of the zonal velocity $u$, we reduce the advectioncondensation system (1) to one spatial dimension, $y$, and write it in Lagrangian form as

$$
\frac{d q(Y, t)}{d t}=-c(Y, t), \frac{d Y(t)}{d t}=V(t)
$$

where $Y(t)$ is the Lagrangian position of an air parcel, and $V(t)=v(Y, t)$ is its Lagrangian velocity. This system describes an air parcel carrying a value of specific humidity, which may be altered by condensation. If the parcel enters a region in which it is supersaturated, $q>$ $q_{s}$, condensation instantaneously reduces $q$ to $q_{s}$. For simplicity, we choose an infinite domain $-\infty<y<\infty$ and assume that the saturation specific humidity $q_{s}(y)$ decreases monotonically with $y$. The specific humidity initial condition is denoted $q_{0}(y)=q(y, 0)$.

The Lagrangian velocities $V(t)$ of air parcels are taken to be independent, identically distributed stochastic processes with stationary statistics, so that the Lagrangian advection-condensation system (3) is a one-dimensional stochastic model. The statistical independence of the parcel velocities neglects spatial correlations that would arise, for example, in an incompressible two-dimensional flow.

Pierrehumbert et al. (2006) used white-noise processes for the Lagrangian velocities. Here we use Orn-
stein-Uhlenbeck processes (Gaussian colored noise) for the Lagrangian velocities. That is, we assume that the Lagrangian velocities have Gaussian statistics with autocorrelation function

$$
\overline{V(t) V\left(t^{\prime}\right)}=\overline{v^{2}} \exp \left(-\left|t-t^{\prime}\right| \tau\right),
$$

where $\tau$ is the Lagrangian velocity correlation time and $\overline{(\cdot)}$ denotes an ensemble average (cf. Gardiner 2004, chapter 3.8). For application to an idealized atmosphere with axisymmetric circulation statistics, the ensemble average may be reinterpreted as a zonal average. The implied stochastic model for Lagrangian parcel trajectories is often called the random flight model and is used to model turbulent dispersion (e.g., van Dop et al. 1985; Thomson 1987, 1990; Pasquero et al. 2001; Sawford 2001).

The velocity at the initial time is assumed to be normally distributed with the same variance $\overline{v^{2}}$ as the stationary velocity distribution, and in general an average over the initial velocity distribution is implied. The displacement probability density function (PDF) associated with an air parcel starting at $Y(0)=y_{0}$ at time zero, given that it is at $Y(t)=y$ at time $t$, is denoted $p\left(y_{0}-y, t\right)$ and is given by the Gaussian density ${ }^{1}$

$$
p(d, t)=\frac{1}{\sqrt{2 \pi L^{2}}} \exp \left(-\frac{d^{2}}{2 L^{2}}\right)
$$

with variance (mean-square displacement)

$$
L(t)^{2}=2 \overline{v^{2}} \tau^{2}\left[\frac{t}{\tau}-1+\exp (-t / \tau)\right]
$$

Since the velocity field is statistically homogeneous and stationary, the displacement PDF depends only on time and position differences.

The specific humidity of an air parcel at $y$ at time $t$ is the minimum of its initial specific humidity $q_{0}$ and the smallest saturation specific humidity $q_{s}$ it encounters between the initial time and $t$. Since $q_{s}$ is monotonically decreasing in $y$, the smallest $q_{s}$ encountered is $q_{s}\left(y_{m}\right)$ where $y_{m}$ is the largest $y$ value encountered, that is, the maximum excursion of the air parcel. Thus, the specific humidity of an air parcel at $Y(t)=y$ at time $t$ is given by

$$
Q\left(y_{0}, y_{m}\right)=\min \left[q_{0}\left(y_{0}\right), q_{s}\left(y_{m}\right)\right],
$$

\footnotetext{
${ }^{1}$ An expression for the conditional displacement PDF given a fixed initial velocity is derived by Uhlenbeck and Ornstein (1930). Averaging over the distribution of initial velocities leads to the displacement PDF (5).
} 
(a)

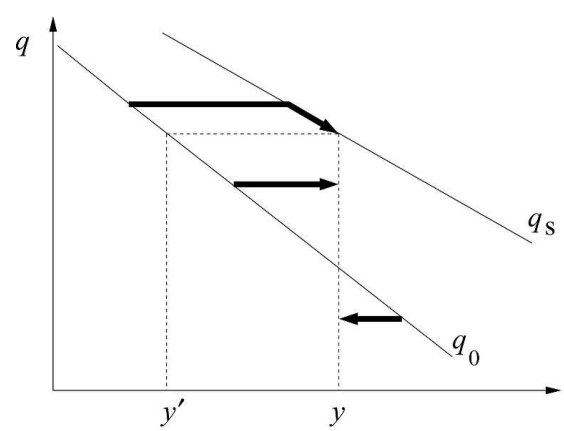

(b)

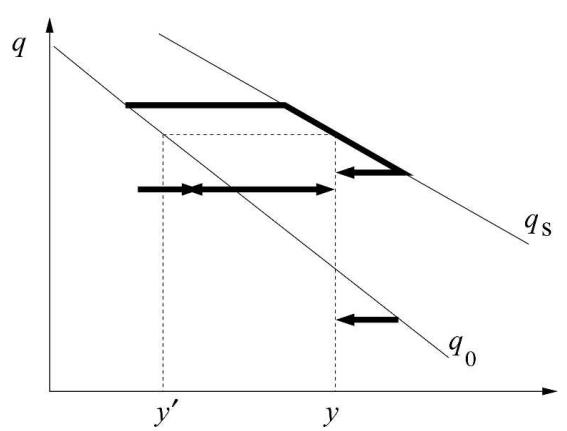

FIG. 1. Sample trajectories (thick solid lines) in one-dimensional stochastic model showing effect of condensation. (a) Trajectories in ballistic limit. (b) More complicated sample trajectories. In each case, three trajectories are shown that end at $y$. The initial specific humidity is the value of $q_{0}$ at the initial position. The specific humidity is conserved except when condensation prevents supersaturation. The point $y^{\prime}$ defined by Eq. (15) is also indicated. Constant gradients in $q_{0}$ and $q_{s}$ are shown for simplicity.

where $Y(0)=y_{0}$ is the initial position of the parcel, $q_{0}\left(y_{0}\right)$ is its initial specific humidity, and $y_{m}$ is the maximum excursion between times zero and $t$ (Pierrehumbert et al. 2006).

To derive a mean evolution equation for the specific humidity, we consider two limits for the correlation time of the Lagrangian velocity: the ballistic limit, $\tau \rightarrow$ $\infty$, and the Brownian limit, $\tau \rightarrow 0 .^{2}$

\section{a. Ballistic limit $(\tau \rightarrow \infty)$}

In the ballistic limit, the Lagrangian velocity correlation time is assumed to be much longer than all other relevant time scales. Lagrangian parcel trajectories evolve ballistically according to the linear relation

$$
Y(t)=y_{0}+V(0) t .
$$

The mean-square displacement $L^{2}(t)=\overline{v^{2}} t^{2}$ increases quadratically with time, as can be seen by expanding (6) for $t / \tau \rightarrow 0$. The displacement PDF (5) simplifies to

$$
p(d, t)=\frac{1}{\sqrt{2 \pi \bar{v}^{2} t^{2}}} \exp \left(-\frac{d^{2}}{2 \overline{v^{2}} t^{2}}\right) .
$$

The displacement PDF (9) may also be directly calculated using the relation (8) between the parcel velocity and position. The generalization to an arbitrary PDF for the velocity would be straightforward.

Figure 1a illustrates sample trajectories in the ballis-

\footnotetext{
${ }^{2}$ We use the term Brownian limit rather than diffusive limit because condensation means that the mean specific humidity need not satisfy a diffusion equation in the limit $\tau \rightarrow 0$.
}

tic limit and the effects of condensation on the specific humidity. Consider a parcel that starts at $Y(0)=y_{0}$ and ends at $Y(t)=y$. If $y_{0}<y$, the maximum excursion $y_{m}$ is equal to the final position $y$, and condensation may occur. If $y_{0}>y$, the maximum excursion $y_{m}$ is equal to the initial position $y_{0}$, and condensation does not occur because the saturation specific humidity decreases monotonically with $y$. Therefore, the final specific humidity (7) for a given trajectory is equal to $Q\left(y_{0}, y\right)$ in the ballistic limit. Averaging over all parcel trajectories that end at $Y(t)=y$, we find the mean specific humidity

$$
\bar{q}(y, t)=\int_{-\infty}^{\infty} d y_{0} p\left(y_{0}-y, t\right) Q\left(y_{0}, y\right) .
$$

From the integral Eq. (10), we can derive the evolution equation for the mean specific humidity $\bar{q}(y, t)$ and expressions for the mean moisture flux $\bar{F}=\overline{v q}$ and the mean condensation rate $\bar{c}$. We take the time derivative of the integral Eq. (10) and use a property of the Gaussian density (9),

$$
\frac{\partial p}{\partial t}=\overline{t v^{2}} \frac{\partial^{2} p}{\partial d^{2}}
$$

which can be viewed as the ballistic limit of the FokkerPlanck equation for the displacement PDF (cf. Gardiner 2004, chapter 5). After some manipulations (see appendix B), we find the mean moisture evolution equation

$$
\frac{\partial \bar{q}}{\partial t}+\frac{\partial \bar{F}}{\partial y}=-\bar{c}
$$


with mean flux and mean condensation rate

$$
\begin{aligned}
& \bar{F}=-\overline{t v^{2}}\left[\frac{\partial \bar{q}}{\partial y}-\frac{d q_{s}}{d y} P\left(y-y^{\prime}, t\right)\right] \\
& \bar{c}=-t \bar{v}^{2} \frac{d q_{s}}{d y} p\left(y-y^{\prime}, t\right)
\end{aligned}
$$

The point $y^{\prime}$ is the point at which the initial specific humidity is equal to the saturation specific humidity at $y$,

$$
q_{s}(y)=q_{0}\left(y^{\prime}\right)
$$

which implies that an air parcel initially at $y^{\prime}$ reaches saturation upon arrival at $y$. For parcels arriving at $y$, the displacement $y-y^{\prime}$ is the minimum signed displacement that must have occurred for condensation at $y$ to be nonzero (see Fig. 1a). For simplicity, we assume that $q_{0}$ is monotonically decreasing in $y$ so that $y^{\prime}$ is uniquely defined, but the analysis can be extended to nonmonotonic $q_{0}$. The function

$$
P(d, t)=\int_{d}^{\infty} d \xi p(\xi, t)
$$

is the complementary cumulative distribution function of parcel displacements; that is, $P(d, t)$ is the probability that a signed displacement greater than $d$ has occurred. Combined with the interpretation of $y-y^{\prime}$ as the minimum displacement for nonzero condensation at $y$, it follows that $P\left(y-y^{\prime}, t\right)$ is the probability of condensation at $y$.

The dependence on the displacement $y-y^{\prime}$ indicates nonlocality in both space and time since $\partial \bar{q}(y, t) / \partial t$ depends on $\bar{q}\left(y^{\prime}, 0\right)$. This is in contrast to the noncondensing limit of the mean moisture evolution Eq. (12),

$$
\frac{\partial \bar{q}}{\partial t}=\overline{t v^{2}} \frac{\partial^{2} \bar{q}}{\partial y^{2}}
$$

which is local in time and space. Nonetheless, the form of the nonlocality in the mean moisture evolution Eq. (12) is remarkably simple. Given that we derived the evolution Eq. (12) from the time rate of change of an integral Eq. (10) for the mean specific humidity, nonlocality was to be expected, and in fact one might have expected an integro-differential equation to result.

For the special initial condition of saturation everywhere, we have $y^{\prime}=y$, and the mean moisture evolution Eq. (12) becomes local in space, although there is still an explicit dependence on the time elapsed, $t$. In general, the initial time $t=0$ plays a distinguished role because at that time all ensemble members share the same instantaneous moisture distribution $q_{0}(y)$. Locality in space for the case of initial saturation is consistent with the fact that all parcels arriving at $y$ having started at $y_{0}<y$ will be saturated at $y$, and thus the evolution equation for the mean moisture at $y$ should not depend on $q_{0}\left(y_{0}\right)$ for $y_{0}<y$.

Since the probability of condensation $P$ is nonnegative, the expression (13) for the mean flux implies that the effect of condensation is to reduce the magnitude of the mean flux for a given mean specific humidity gradient. If the mean specific humidity at point $y_{s}$ is equal to the saturation specific humidity, $\bar{q}\left(y_{s}, t\right)=q_{s}\left(y_{s}\right)$, the gradients must also be equal there, $\partial \bar{q}\left(y_{s}, t\right) / \partial y=$ $d q_{s}\left(y_{s}\right) / d y$, so that supersaturation does not occur in the neighborhood of $y_{s}$. At such a point, $y^{\prime}=y$ and $P\left(y^{\prime}-\right.$ $y)=0.5$, and the mean flux (13) is $-0.5 t \overline{v^{2}} \partial \bar{q} / \partial y$, exactly one-half the flux for a noncondensing passive scalar with the same mean gradient. However, this result must be understood in a limiting sense, since mean saturation can only occur at the initial time, $t=0$, when the moisture flux in both the condensing and noncondensing cases is zero in the ballistic limit.

\section{b. Brownian limit $(\tau \rightarrow 0)$}

In the Brownian limit, the Lagrangian velocity correlation time is assumed to be much shorter than all other relevant time scales. The Brownian limit is the limit of the correlation time $\tau$ approaching zero with the integral $D=\int_{t}^{\infty} \overline{V(t) V\left(t^{\prime}\right)} d t^{\prime}=\overline{v^{2}} \tau$ of the autocorrelation function (4) held constant (cf. Gardiner 2004, chapter 6.5). In this limit, the Lagrangian velocity is a Gaussian white-noise process with autocorrelation function

$$
\overline{V(t) V\left(t^{\prime}\right)}=2 D \delta\left(t-t^{\prime}\right)
$$

A noncondensing passive scalar advected by this velocity field diffuses with diffusivity $D$. The mean-square displacement $L^{2}=2 D t$ increases linearly with time, and the displacement PDF (5) simplifies to the diffusion kernel

$$
p(d, t)=\frac{1}{\sqrt{4 \pi D t}} \exp \left(-\frac{d^{2}}{4 D t}\right)
$$

Pierrehumbert et al. (2006) showed how to solve an initial value problem for the advection-condensation system in this Brownian limit when the initial condition for the specific humidity is saturated everywhere. We will derive the evolution equation for the mean specific humidity in the more general case of arbitrary initial conditions. 
Unlike in the ballistic limit, in the Brownian limit the velocity can change sign along a trajectory, so we need to consider more complicated trajectories (see Fig. 1b). We denote by $p_{\max }\left(y_{0}, y_{m} \mid y, t\right)$ the conditional PDF associated with a trajectory starting at $Y(0)=y_{0}$, with maximum excursion $y_{m}$ between times zero and $t$, given that the trajectory ends at $Y(t)=y$. Averaging over all trajectories ending at $y$ at time $t$ leads to the mean specific humidity

$$
\bar{q}(y, t)=\int_{-\infty}^{\infty} d y_{0} \int_{-\infty}^{\infty} d y_{m} p_{\max }\left(y_{0}, y_{m} \mid y, t\right) Q\left(y_{0}, y_{m}\right),
$$

with the final specific humidity $Q$ for a given trajectory given by expression (7).

The calculation of the maximum excursion $\mathrm{PDF} p_{\text {max }}$ is similar to the first-passage time problem of calculating how long a parcel advected by a stochastic velocity field remains in a specified region (cf. Gardiner 2004, chapter 5.2). It is shown in appendix A that

$p_{\max }\left(y_{0}, y_{m} \mid y, t\right)= \begin{cases}-2 p^{\prime}\left(2 y_{m}-y-y_{0}, t\right) & \text { if } y_{m}>y, y_{0} \\ 0 & \text { otherwise }\end{cases}$

where $p^{\prime}(d, t)$ denotes the derivative of the displacement PDF $p(d, t)$ with respect to its spatial argument. The displacement PDF $p\left(y-y_{0}, t\right)$ is associated with trajectories starting at $y_{0}$ and ending at $y$ a time $t$ later, regardless of their maximum excursion. It can be verified that integration of the maximum excursion PDF (21) over all maximum excursions $y_{m}$ yields the displacement PDF. Integration over the initial position $y_{0}$ yields the PDF of maximum excursion regardless of initial position discussed by Pierrehumbert et al. (2006).

In a similar manner as in the ballistic limit, we can derive the evolution equation for the mean specific humidity from the integral Eq. (20) for the mean specific humidity. Taking the time derivative of the integral Eq. (20) and using the Fokker-Planck equation for the diffusion kernel,

$$
\frac{\partial}{\partial t} p(d, t)=D \frac{\partial^{2}}{\partial d^{2}} p(d, t)
$$

leads to the mean moisture evolution Eq. (12) with mean flux and mean condensation rate (cf. appendix B)

$$
\bar{F}=-D \frac{\partial \bar{q}}{\partial y}
$$

$$
\bar{c}=-2 D \frac{d q_{s}}{d y} p\left(y-y^{\prime}, t\right) .
$$

The point $y^{\prime}$ is again defined by (15), and the analysis can again be extended to the case in which the initial condition $q_{0}(y)$ is nonmonotonic and $y^{\prime}$ is not unique. Note that the mean moisture flux cannot be defined in the usual way as an average $\overline{v q}$ of velocity and specific humidity fluctuations because the instantaneous velocity is not defined in this Brownian limit.

As in the ballistic limit, the evolution equation is nonlocal, but the nonlocality takes a simple form. The mean condensation rate (24) has a similar form to the mean condensation rate (14) in the ballistic limit. The mean moisture flux (23) has the same form as for ordinary diffusion of a noncondensing scalar, as one might have expected given that the velocity has no memory.

\section{c. Initial value problem}

To illustrate features of the stochastic model, we consider an initial value problem. The saturation specific humidity is taken to be exponentially decaying in $y$ over the length scale $L_{s}$, and the initial specific humidity is taken to be subsaturated and exponentially decaying over the same length scale $L_{s}$,

$$
q_{s}=\hat{q} \exp \left(-\frac{y}{L_{s}}\right), q_{0}=\hat{q} \exp \left(-\frac{y+m}{L_{s}}\right),
$$

where $\hat{q}$ is a characteristic specific humidity and the length scale $m \geq 0$ determines the degree of initial subsaturation.

We compare the mean specific humidities after an evolution time $T$ in the ballistic limit, in the Brownian limit, and in the case of finite velocity correlation time $\tau=\mathrm{T} / 8$. The evolution time $T$ in each case is chosen to be the time at which the rms displacement $L(T)$ is equal to $4 L_{s}$. The initial subsaturation length scale is chosen as $m=L_{s} / 4$. Numerical simulations were carried out for all three cases. ${ }^{3}$

Analytic expressions for the mean specific humidity $\bar{q}(y, t)$ were found in the ballistic and Brownian limits by substituting the saturation and initial specific humidities (25) into the integral formulas (10) and (20). The result for the ballistic limit is

\footnotetext{
${ }^{3}$ The stochastic simulations each used 200000 particles in the domain $\left(-8 L_{s}, 8 L_{s}\right)$. The particles were initially uniformly distributed in the domain, with initial specific humidity (25). The mean specific humidity distributions at the end of the simulations were calculated using averages over the particles in 50 uniformly spaced grid cells. The velocities in the case with finite velocity correlation time were generated using the method described by Bartosch (2001).
} 


$$
\bar{q}(y, t)=\frac{\hat{q}}{2} \exp \left(-\frac{y}{L_{s}}\right)\left[\exp \left(\frac{L^{2}-2 L_{s} m}{2 L_{s}^{2}}\right) \operatorname{erfc}\left(\frac{L^{2}-L_{s} m}{\sqrt{2} L L_{s}}\right)+\operatorname{erfc}\left(\frac{m}{\sqrt{2} L}\right)\right],
$$

where $L^{2}(t)=\bar{v}^{2} t^{2}$, and for the Brownian limit,

$$
\bar{q}(y, t)=\frac{\hat{q}}{2} \exp \left(-\frac{y}{L_{s}}\right)\left[\exp \left(\frac{L^{2}-2 L_{s} m}{2 L_{s}^{2}}\right) \operatorname{erfc}\left(\frac{L^{2}-L_{s} m}{\sqrt{2} L L_{s}}\right)+\exp \left(\frac{L^{2}+2 L_{s} m}{2 L_{s}^{2}}\right) \operatorname{erfc}\left(\frac{L^{2}+L_{s} m}{\sqrt{2} L L_{s}}\right)\right]
$$

where $L^{2}(t)=2 D t$ and erfc is the complementary error function. These analytical expressions satisfy the mean moisture evolution equations derived in the previous two subsections, as can be verified by substitution. A comparison of the second terms in brackets in expressions (26) and (27) shows that the mean specific humidity in the Brownian limit is always less than or equal to that in the ballistic limit for equal spreading length $L$, regardless of the values of the other parameters. In general, one expects greater condensation in the Brownian limit than in the ballistic limit for equal $L$, given that the maximum excursions of parcel trajectories are greater in the Brownian limit for given start and end points.

Figure 2 shows the results, the normalized mean specific humidities $\bar{q}(y, T) / \hat{q}$ as functions of $y / L_{s}$. The exact and numerical results for the Brownian and ballistic limits are in good agreement. In all cases, throughout the domain the mean condensation rate is nonzero, and the mean specific humidity is subsaturated. At the times shown, there is positive mean moisture flux convergence, but the mean condensation rate is greater, and the mean specific humidities are decreasing.

In the case of finite velocity correlation time, the ratio of evolution time to correlation time is quite large $(T / \tau=8)$, but Fig. 2 shows that the resulting mean specific humidity is not well approximated by the Brownian limit because of the effects of condensation. In considering the relative magnitude of the correlation time, one must take into account the time scales introduced by condensation. For example, the time scale $\tau_{s}$ over which a parcel drawn from the initial condition reaches saturation is given by setting the spreading length $L$ equal to the distance to saturation $m$ in Eq. (6). For the parameters used above, $\tau_{s} / \tau=0.24$, and so the Brownian limit $\tau \rightarrow 0$ is not appropriate.

Although the ballistic and Brownian limits discussed in this section illustrate how condensation affects the kinematics of a moisture tracer, a finite Lagrangian velocity correlation time is more appropriate for atmospheric flows. In the next section, we discuss a case with a finite correlation time and an evaporation source.

\section{Two-dimensional turbulence}

The advection-condensation system (1) with a twodimensional turbulent velocity field provides a closer representation of the kinematics of moisture transport and condensation on isentropes than the one-dimensional stochastic model of the previous section. We focus on the mean condensation rate and mean moisture flux in homogeneous two-dimensional turbulence with an imposed constant gradient in mean specific humidity. Turbulent advection of specific humidity down the imposed mean gradient generates moisture variance, which cascades to small scales. This nonlinear cascade of moisture variance in spectral space corresponds to the generation of filamentary structures in physical space, a process that can be expected to be modified by condensation.

\section{a. Velocity field}

We consider an incompressible velocity field which is statistically steady, homogeneous, and isotropic. The domain is a square of side $2 \pi$ with doubly periodic boundary conditions. The velocity field is simulated with a dealiased pseudospectral code at $128^{2}$ resolution, which integrates the vorticity equation

$$
\frac{\partial \zeta}{\partial t}+J(\psi, \zeta)=-r \zeta+\mathcal{F}+\mathcal{G}
$$

with streamfunction $\psi$, vorticity $\zeta=\nabla^{2} \psi$, and Jacobian operator $J$. The parameter $r$ is a constant Rayleigh drag coefficient, $\mathcal{F}$ is a small-scale enstrophy filter representing subgrid-scale processes, and $\mathcal{G}$ is a large-scale forcing term.

The forcing $\mathcal{G}$ is by a random Markov process, localized at wavenumbers $2 \leq k \leq 4$ where $\mathbf{k}$ is the twodimensional wavenumber, $k=\|\mathbf{k}\|$, and the minimum wavenumber is $k=1$. The enstrophy filter $\mathcal{F}$ is an ex- 


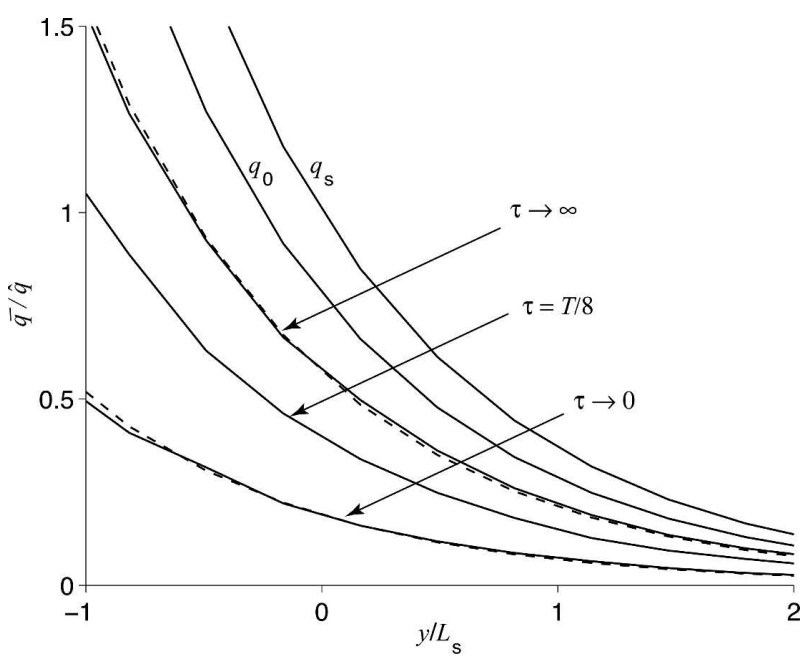

FIG. 2. Mean specific humidity vs meridional distance for initial value problem. Moisture distributions are shown after the evolution times $T$ at which $L(T)=4 L_{s}$ in each case. Solid lines are shown in order of decreasing magnitude: saturation specific humidity, specific humidity initial condition, and simulations for ballistic limit $(\tau \rightarrow \infty)$, for finite velocity correlation time $(\tau=T / 8)$, and for Brownian limit $(\tau \rightarrow 0)$. Dashed lines represent the exact solutions (26) and (27) for the ballistic and Brownian limits.

ponential cutoff filter, with parameters chosen such that the filter is only active for wavenumbers $k \geq 50$. Details of the forcing and enstrophy filter are described by Smith et al. (2002).

Results are nondimensionalized using an integral wavenumber $k_{e}$ and an eddy time scale $\tau_{e}$. The integral wavenumber is the first moment $k_{e}=\int d k k E(k) / \int$ $d k E(k)$ of the energy spectrum $E(k)$; its numerical value is $k_{e}=2.45$ in the simulations. The eddy time scale is based on the enstrophy, $\tau_{e}=2 \pi /\left(\overline{\zeta^{2}}\right)^{1 / 2}$, where $\overline{(\cdot)}$ now denotes a space and time average. The eddy time scale will be used as the reference time scale to describe the simulations. The Rayleigh drag coefficient is $r=0.35 / \tau_{e}$, which is sufficiently large to inhibit a significant inverse energy cascade. As is often found, the energy spectrum in the inertial range is somewhat steeper than the $k^{-3}$ power law that would be expected in a pure enstrophy cascade (Oetzel and Vallis 1997; Gotoh 1998); it is closer to $k^{-3.5}$. Unlike many studies of two-dimensional turbulence, we focus on the forward enstrophy cascade rather than the inverse energy cascade since there appears to be no significant inverse energy cascade in the earth's atmosphere (Boer and Shepherd 1983; Schneider and Walker 2006).

\section{b. Moisture field}

The moisture field is advected by the two-dimensional velocity field and evolves according to

$$
\frac{\partial q}{\partial t}+J(\psi, q)=e-c+\mathcal{F},
$$

where $e$ is a spatially and temporally constant evaporation rate, and $\mathcal{F}$ is the same small-scale filter as in the vorticity Eq. (28). The condensation rate is given by

$$
c=\frac{1}{\tau_{c}}\left(q-q_{s}\right) H\left(q-q_{s}\right),
$$

where $H$ is the Heaviside step function, and the limit $\tau_{c} \rightarrow 0$ is taken so that supersaturation does not occur. The condensation term is treated implicitly in the numerical time-stepping scheme.

The mean specific humidity and the saturation specific humidity have the same constant mean gradient $\gamma$, a stipulation that ensures that specific humidity fluctuations $q^{\prime}$ are statistically homogeneous. That is, moisture fields are decomposed as

$$
\begin{aligned}
q & =\langle q\rangle+\gamma y+q^{\prime}, \\
q_{s} & =\gamma y,
\end{aligned}
$$

where $\langle\cdot\rangle$ denotes an average over the domain, and the fluctuation $q^{\prime}$ satisfies periodic boundary conditions. The spatially averaged saturation specific humidity $\left\langle q_{s}\right\rangle$ is taken to be zero, and so the spatially averaged specific humidity $\langle q\rangle$ is negative or zero. Taking the spatial average of the moisture Eq. (29) gives

$$
\frac{d\langle q\rangle}{d t}=e-\langle c\rangle,
$$

since the small-scale filter $\mathcal{F}$ has no effect on $\langle q\rangle$, and from this it follows that the equation for the moisture fluctuation $q^{\prime}$ is

$$
\frac{\partial q^{\prime}}{\partial t}+J\left(\psi, q^{\prime}\right)+\frac{\partial \psi}{\partial x} \gamma=\langle c\rangle-c+\mathcal{F}
$$

The equation for the moisture fluctuation is statistically homogeneous since the condensation rate $c$ depends only on $q-q_{s}=\langle q\rangle+q^{\prime}$ with no explicit dependence on the meridional coordinate $y$. The homogeneity makes this formulation of moisture kinematics similar to the lower layer of the two-layer quasigeostrophic model of Lapeyre and Held (2004).

We will analyze statistically steady states of the simulations. In a statistically steady state, according to Eq. (33), evaporation balances the spatially and temporally averaged condensation, $\bar{c}=e$, with $\overline{(\cdot)}$ denoting the space and time average. We will discuss the mean saturation deficit $\bar{q}_{s}-\bar{q}=-\bar{q}$, a measure of the degree of subsaturation, and the mean moisture flux $\overline{v q}$ down the imposed mean gradient, with $v=\partial \psi / \partial x$ denoting the velocity component parallel to the mean specific hu- 
(a)

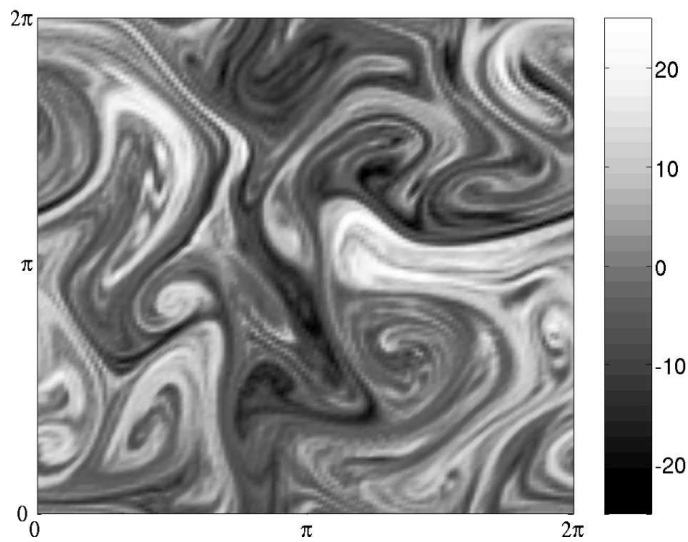

(b)

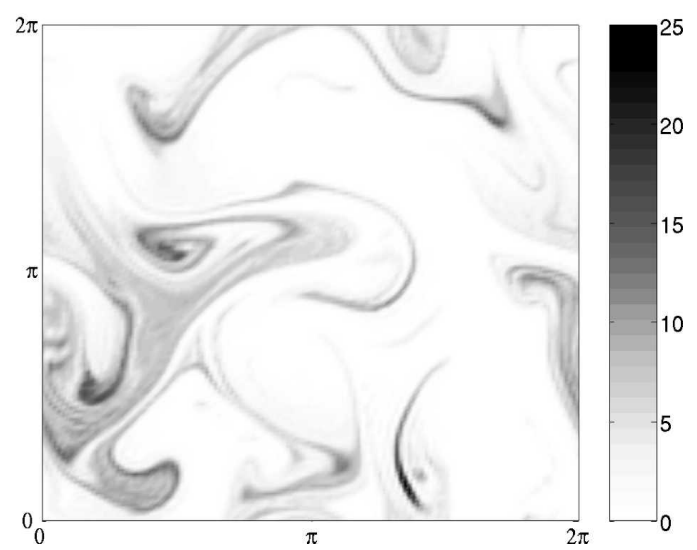

FIG. 3. Snapshots of moisture fields in two-dimensional turbulence simulations. (a) Specific humidity fluctuation $q^{\prime} k_{e} /|\gamma|$ in simulation without evaporation and condensation. (b) Saturation deficit $\left(q_{s}-q\right) k_{e} /|\gamma|$ in simulation with condensation and evaporation $e=1.45|\gamma| /\left(k_{e} \tau_{e}\right)$. Saturation occurs where the saturation deficit is zero. The moisture fluctuation and saturation deficit fields are statistically homogeneous, and both snapshots are from statistically steady states of the simulations. The mean specific humidity gradient in both cases is directed down the page.

midity gradient. The choice of the imposed mean moisture gradient $\gamma$ sets the moisture scale, and our results are nondimensionalized using $\gamma$.

Figure 3a shows a snapshot of the specific humidity fluctuation $q^{\prime}$ at an instant after a statistically steady state has been reached in a simulation with no evaporation or condensation. In this simulation, specific humidity is a conventional passive scalar with characteristic filamentary structures. Figure $3 \mathrm{~b}$ shows a snapshot of the saturation deficit $q_{s}-q=-\left(\langle q\rangle+q^{\prime}\right)$, also after statistically steady state has been reached, in a simulation with condensation and evaporation $e=1.45|\gamma| /$ $\left(k_{e} \tau_{e}\right)$. Large regions can be seen to be close to saturation, reminiscent of tongues of high relative humidity air moving poleward in the earth's atmosphere, interlaced with filaments with large saturation deficits.

\section{c. Simulations and results}

We performed a series of ten simulations with a different evaporation rate for each simulation. The mean moisture flux $\overline{v q}$, mean condensation rate $\bar{c}$, and mean saturation deficit $-\bar{q}$ at steady state were calculated for each simulation. The time average is over approximately 475 eddy time scales $\tau_{e}$. In simulations with small evaporation rates, condensation has little effect and condensation events are rare, making long averaging times necessary.

Figure 4 shows the mean condensation rate as a function of the mean saturation deficit. As might be expected, the mean condensation rate is a decreasing function of mean saturation deficit. For large mean saturation deficits, condensation events are rare, and the mean condensation rate tends to zero. For large evaporation rates, the mean saturation deficit tends to zero as the evaporation overpowers the ability of the turbulence to generate subsaturated fluid parcels through advection up the mean moisture gradient.

Figure 5 shows the mean moisture flux as a function of the mean saturation deficit. The flux is zero at zero saturation deficit and increases with increasing saturation deficit. For large saturation deficit, the flux asymptotes to the no-condensation value of $\overline{v q} k_{e}^{2} \tau_{e} / \gamma=5.91$. Thus, as was found in the ballistic limit of the onedimensional stochastic model, the effect of condensa-

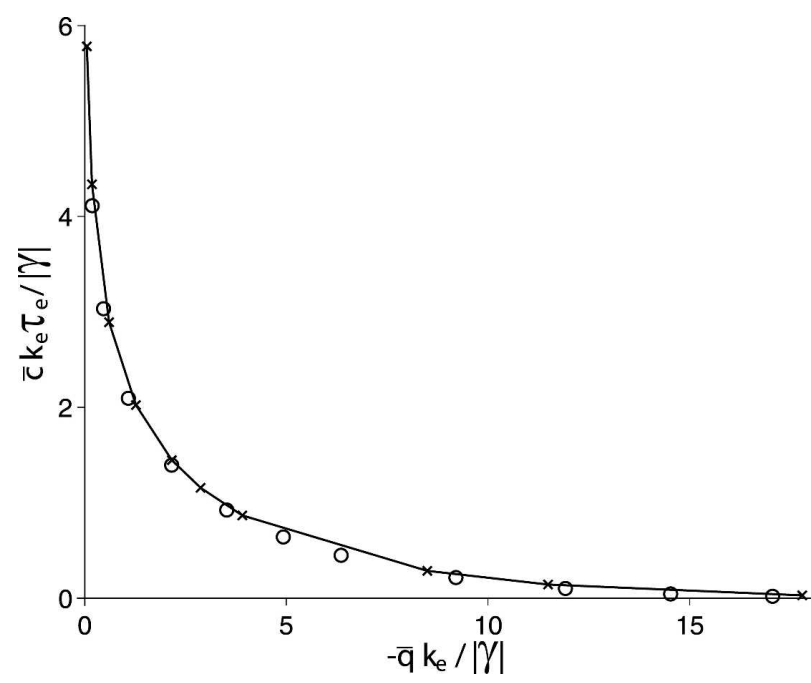

FIG. 4. Mean condensation rate vs mean saturation deficit in two-dimensional turbulence simulations (solid line and crosses) and in one-dimensional stochastic model (open circles). 


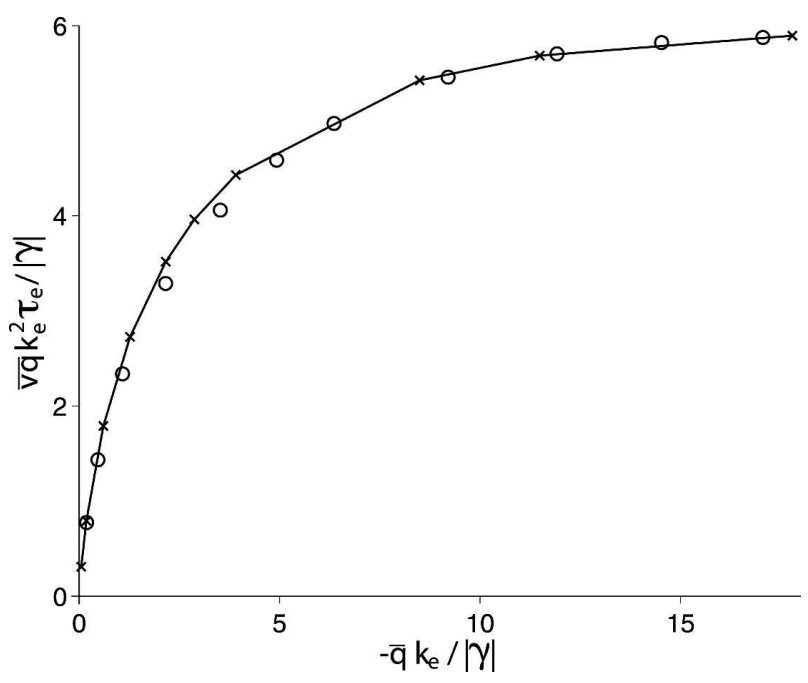

FIG. 5. Mean moisture flux vs mean saturation deficit in twodimensional turbulence simulations (solid line and crosses) and in one-dimensional stochastic model (open circles).

tion is to reduce the mean moisture flux for a given mean moisture gradient, that is, to reduce the effective eddy diffusivity. This is also consistent with the effect of condensation on the moisture flux in the quasigeostrophic simulations of a moisture tracer without latent heating described by Lapeyre and Held (2004). In the limit of small mean saturation deficit, the fluctuation variance $\left\langle q^{\prime 2}\right\rangle$ tends to zero because $q \leq q_{s}$ everywhere, and so the mean moisture flux also tends to zero. (In the more realistic case of fluctuating saturation specific humidity, the specific humidity flux in the limit of small mean saturation deficit would be equal to the flux of saturation specific humidity.)

That the mean moisture flux in the turbulence simulations tends to zero for small mean saturation deficit may be compared with the results derived in section 3 for the stochastic model. In the ballistic limit, in the absence of an evaporation source, mean saturation was only possible at time zero when both condensing and noncondensing scalars have zero flux. In the Brownian limit, the stochastic model gave a flux that was independent of the mean saturation deficit. A more general formulation of the stochastic model is now considered, allowing comparison with the two-dimensional simulations by including evaporation and a finite Lagrangian velocity time.

\section{d. Comparison with stochastic model}

It is difficult to find analytical solutions for mean moisture fields using the one-dimensional stochastic model of section 3 with a finite velocity correlation time and with an evaporation source. Apart from the diffi- culty of including an evaporation source, we would need to calculate the maximum excursion PDF $p_{\max }$ at finite velocity correlation time. The task of calculating the maximum excursion PDF for Ornstein-Uhlenbeck velocities with finite correlation time is related to the first-passage time problem for a process driven by Ornstein-Uhlenbeck noise, for which no simple analytical solution is known (e.g., Hagan et al. 1989).

Instead, we simulate the stochastic processes numerically. We solve an advection-condensation problem analogous to the two-dimensional turbulence simulations, with the same constant gradients in mean specific humidity and saturation specific humidity, and with periodic boundary conditions and a constant evaporation rate, but in one spatial dimension. The numerical simulation is similar to the simulation with finite velocity correlation time described in section 3, except for the use of periodic boundary conditions and an evaporation source, and the fact that because of statistical homogeneity, averages may now be taken over the entire domain.

One further property of the moisture tracer in the two-dimensional turbulence simulations that must be taken into account is the combined effect of the nonlinear cascade and dissipation by the filter representing subgrid-scale processes. The moisture variance dissipation in the turbulence simulations is due to both condensation and the small-scale filter, with the small-scale filter becoming relatively more important for larger mean saturation deficits, for which condensation events become rare. The dissipation of moisture variance $\left\langle q^{\prime 2}\right\rangle / 2$ due to condensation can be written in terms of the spatially averaged saturation deficit $-\langle q\rangle$ and condensation rate $\langle c\rangle$ as

$$
\left\langle c q^{\prime}\right\rangle=-\langle c\rangle\langle q\rangle,
$$

where we have used the fact that condensation only occurs where there is instantaneous saturation. The small-scale filter contribution is difficult to represent in the stochastic model given that the model is onedimensional and based on a Lagrangian formulation. Moisture variance is important, however, because the frequency of condensation events is influenced by the magnitude of moisture fluctuations. We crudely represent small-scale moisture variance dissipation in the stochastic model by setting $q^{\prime}(y, 0)=0$ everywhere initially and computing the spatially averaged condensation rate and moisture flux after an irreversible mixing time $\tau_{i}$. The variance after time $\tau_{i}$ is expected to be approximately equal to the steady-state variance in the turbulence simulations. Thus we solve a specific initial value problem to estimate statistics in the steady states of the turbulence simulations. We choose for $\tau_{i}$ the time 
scale of variance generation and dissipation for a noncondensing passive scalar $\phi$ with mean gradient $\gamma$ in the statistically steady state of the turbulence simulations,

$$
\tau_{i}=-\frac{\overline{\phi^{\prime 2}}}{2 \overline{v \phi} \gamma}
$$

The time scale $\tau_{i}$ is independent of the magnitude of the mean gradient $\gamma$. In our simulations $\tau_{i}=4.35 \tau_{e}$.

To avoid associating a particular evaporation rate with a given initial mean saturation deficit a priori, we employ an iterative procedure. For a given initial mean saturation deficit, we pick a test evaporation rate and evolve the stochastic model over time $\tau_{i}$. We then iteratively repeat the simulation using the mean condensation rate at time $\tau_{i}$ from the last simulation as the new evaporation rate, until the evaporation rate and the mean condensation rate at time $\tau_{i}$ converge. Repeating this procedure for a set of different initial mean saturation deficits, we obtain the mean moisture flux and mean condensation rate at time $\tau_{i}$ as functions of the mean saturation deficit at time $\tau_{i}$. All statistics (including the mean saturation deficit) are taken at time $\tau_{i}$, since it is then that the mean condensation rate is consistent with the evaporation rate, and the moisture variance is consistent with the variance in the turbulence simulations. The moisture variance at time $\tau_{i}$ and the steady-state moisture variance in the turbulence simulations agree to within $30 \%$ in all cases, justifying the choice (36) of the variance dissipation time. The value of $\tau_{i}$ used was doubled in a sensitivity test, with the result that the moisture flux agreed with the turbulence simulation values to within $15 \%$, but the mean condensation rate for large mean saturation deficit was too great by up to a factor of 2 .

We match the Lagrangian velocity variance $\overline{v^{2}}$ in the stochastic model to the variance of one component of the Eulerian velocity in the two-dimensional turbulence simulations. We choose the Lagrangian velocity correlation time $\tau$ such that the eddy diffusivity of a noncondensing passive scalar in the turbulence simulations is equal to that of the stochastic model at time $t=\tau_{i}$ without condensation, $\overline{v^{2}} \tau\left[1-\exp \left(-\tau_{i} / \tau\right)\right]$, which yields $\tau=0.42 \tau_{e}$. The ratio of variance dissipation time to Lagrangian correlation time is $\tau_{i} / \tau=10.3$; hence, the diffusivities of the stochastic process without condensation are virtually identical at $t=\tau_{i}$ and in the long time limit. As discussed in section 3c, although the velocity correlation time is much smaller than the mixing time $\tau_{i}$, the Brownian limit $\tau \rightarrow 0$ is not appropriate for the moisture tracer because of the effects of condensation.

Figures 4 and 5 show a comparison of the two- dimensional turbulence mean condensation rate and mean moisture flux with the results from the onedimensional stochastic model. The correspondence is good, suggesting that the stochastic model captures fundamental effects of condensation in the turbulence simulations.

\section{Conclusions}

We have studied the dependence of the mean moisture flux and mean condensation rate on other mean field statistics in a one-dimensional stochastic model and in a two-dimensional turbulent flow.

For the stochastic model, we have found analytic expressions for the mean flux and condensation rate in the Brownian and ballistic limits of small and large Lagrangian velocity correlation time. These expressions, derived using averages over Lagrangian trajectories, are nonlocal in space and time, but the functional form of the nonlocality is simple and does not involve integral expressions.

The dependence of the mean moisture flux and mean condensation rate on mean saturation deficit in the two-dimensional simulations was reproduced with the one-dimensional stochastic model. The stochastic model was used to solve an initial value problem in which an initial state without moisture fluctuations evolved over a variance dissipation time. At the variance dissipation time, the statistics in the stochastic model were taken to be representative of the statistically steady states of the turbulence simulations. With this procedure, we were able to capture the effect of moisture variance dissipation by the small-scale filter in the turbulence simulations.

A general result from the stochastic model and from the two-dimensional turbulence simulations is that condensation reduces the mean moisture flux for a given mean moisture gradient compared with the mean flux of a noncondensing scalar, except in the Brownian limit in which the moisture flux is unchanged by condensation. The mean moisture flux was found to decrease with decreasing mean saturation deficit in the twodimensional simulations and in the stochastic model, except in the Brownian limit. The mean condensation rate was found to increase with decreasing mean saturation deficit in the two-dimensional simulations and in the stochastic model. If these results also hold for tropospheric eddy moisture transport, they could imply a stabilizing influence on the extratropical relative humidity in that higher relative humidity would lead to greater condensation, a smaller moisture flux, and possibly less flux convergence, and vice versa. It will be important to determine if the dependence of the mean 
moisture flux on mean saturation deficit is significantly different in turbulence with a planetary vorticity gradient and thus with Rossby waves.

The Brownian limit of small velocity correlation time is often a useful approximation when considering the turbulent dispersion of a noncondensing tracer over many eddy turnover times. For a condensing moisture tracer, however, one must also compare the velocity correlation time with time scales related to condensation, such as a time-to-saturation time scale. Since these condensation time scales can be small compared with the velocity correlation time, the Brownian limit will often not be appropriate for a moisture tracer even when it is appropriate for a noncondensing tracer. For example, air parcels carried poleward along an isentrope from the subtropics to higher latitudes in a typical baroclinic eddy typically reach saturation within times of order one eddy turnover time, within which the meridional velocity typically is of one sign (poleward), indicating that the Brownian limit would not be appropriate to describe this process.

Possible future work includes the examination of inhomogeneous steady states and allowing for fluctuations in saturation specific humidity and for a more wavelike advecting velocity field. Comparisons could then be made between the stochastic model and moisture kinematics on isentropes in general circulation models and observations, with the general aim of understanding the relative humidity distribution and poleward moisture transport, and how they can change as the climate changes.

Acknowledgments. We are grateful for support by the Davidow Discovery Fund, by an Alfred P. Sloan Research Fellowship, and by the National Science Foundation (Grant ATM-0450059). The pseudospectral code used in section 4 is a modified version of the spectral quasigeostropic model developed by Shafer Smith. We gratefully acknowledge helpful suggestions from James Gleeson, Raymond Pierrehumbert, and an anonymous referee.

\section{APPENDIX A}

\section{Maximum Excursion Statistics in Brownian Limit}

Here we derive expression (21) for the maximum excursion PDF $p_{\max }\left(y_{0}, y_{m} \mid y, t\right)$ associated with a trajectory starting at $Y(0)=y_{0}$, with maximum excursion $y_{m}$ between times zero and $t$, given that the trajectory ends at $Y(t)=y$. The stochastic process for parcel velocities is a Gaussian white noise process with autocorrelation function (18).
We start by calculating the PDF $\mathcal{P}\left(y_{0}, y_{m}, t_{0} \mid y, t\right)$ associated with a trajectory starting at $Y\left(t_{0}\right)=y_{0}$, without crossing $y_{m}$ between times $t_{0}$ and $t$, given that it ends at $Y(t)=y$. This can be found by solving the adjoint of the Fokker-Planck Eq. (22),

$$
-\frac{\partial \mathcal{P}}{\partial t_{0}}=D \frac{\partial^{2} \mathcal{P}}{\partial y_{0}^{2}}
$$

with final condition $\mathcal{P}\left(y_{0}, y_{m}, t \mid y, t\right)=\delta\left(y-y_{0}\right)$ and absorbing boundary condition $\mathcal{P}\left(y_{m}, y_{m}, t_{0} \mid y, t\right)=0$. The absorbing boundary condition imposes the condition that parcels must not cross $y_{m}$ (see Gardiner 2004, chapter 5.2). The solution is found in terms of the displacement PDF $p(d, t)$ given by Eq. (19) using the method of image sources (see, e.g., Morse and Feshbach 1953). The image solution is chosen so that the absorbing boundary condition is satisfied, and is centered at $\hat{y}=2 y_{m}-y$,

$$
\mathcal{P}\left(y_{0}, y_{m}, t_{0} \mid y, t\right)=p\left(y-y_{0}, t-t_{0}\right)-p\left(\hat{y}-y_{0}, t-t_{0}\right),
$$

where $y_{0}, y<y_{m}$.

The probability of parcels reaching the interval $\left[y_{m}\right.$ $-d y_{m}, y_{m}$ ] but not crossing $y_{m}$ is $\partial \mathcal{P} / \partial y_{m} d y_{m}$. The PDF associated with just reaching $y_{m}$, rather than not crossing $y_{m}$, is then

$$
\begin{aligned}
p_{\max }\left(y_{0}, y_{m} \mid y, t-t_{0}\right) & =\partial \mathcal{P} / \partial y_{m} \\
& =-2 p^{\prime}\left(2 y_{m}-y-y_{0}, t-t_{0}\right),
\end{aligned}
$$

where $p^{\prime}$ denotes the derivative of $p(d, t)$ with respect to its spatial argument. Equation (21) for the maximum excursion PDF follows by setting $t_{0}=0$.

\section{APPENDIX B}

\section{Derivation of Mean Moisture Evolution Equation}

\section{a. Ballistic limit}

Taking the time derivative of the ballistic integral Eq. (10) for the mean specific humidity and using the Fokker-Planck Eq. (11) for the displacement $\operatorname{PDF} p(d, t)$ leads to

$$
\frac{\partial}{\partial t} \bar{q}(y, t)=\overline{t v^{2}} \int_{-\infty}^{\infty} d y_{0}\left[\frac{\partial^{2}}{\partial y^{2}} p\left(y_{0}-y, t\right)\right] Q\left(y_{0}, y\right) .
$$

Substituting for the integral on the right-hand side by calculating $\partial^{2} \bar{q} / \partial y^{2}$ from the integral Eq. (10) yields 
$$
\frac{\partial \bar{q}}{\partial t}=t v^{2} \frac{\partial^{2} \bar{q}}{\partial y^{2}}-t v^{2} \int_{-\infty}^{\infty} d y_{0}\left[\frac{\partial p}{\partial y} \frac{\partial Q}{\partial y}+\frac{\partial}{\partial y}\left(p \frac{\partial Q}{\partial y}\right)\right],
$$

with $\bar{q}=\bar{q}(y, t), p=p\left(y_{0}-y, t\right)$, and $Q=Q\left(y_{0}, y\right)$. Expressing the specific humidity $Q$ defined by (7) with the Heaviside step function $H$ as

$$
Q\left(y_{0}, y\right)=q_{0}\left(y_{0}\right)+\left[q_{s}(y)-q_{0}\left(y_{0}\right)\right] H\left[q_{0}\left(y_{0}\right)-q_{s}(y)\right],
$$

and using that the distributional derivative of the Heaviside step function is the Dirac delta function, we obtain the mean evolution Eq. (12) with flux (13) and condensation rate (14).

The identification of the mean flux and mean condensation rate terms can be verified by direct calcula- tion. For example, the condensation rate for a parcel at $Y(t)=y$ at time $t$ that started at $Y(0)=y_{0}$ at time zero is

$$
-v \frac{d}{d y} q_{s}(y)=-\frac{\left(y-y_{0}\right)}{t} \frac{d}{d y} q_{s}(y)
$$

if $y_{0}<y^{\prime}$ and zero otherwise, where $y^{\prime}$ is defined by (15). The mean condensation rate (14) can then be calculated by averaging over initial positions

$$
\bar{c}(y, t)=-\int_{-\infty}^{y^{\prime}} d y_{0} p\left(y-y_{0}, t\right) \frac{\left(y-y_{0}\right)}{t} \frac{d}{d y} q_{s}(y) .
$$

\section{b. Brownian limit}

Taking the time derivative of the Brownian integral Eq. (20) for the mean specific humidity and using the Fokker-Planck Eq. (22) for the displacement PDF $p(d, t)$ leads to

$$
\frac{\partial}{\partial t} \bar{q}(y, t)=2 D \int_{-\infty}^{\infty} d y_{0} \int_{-\infty}^{\infty} d y_{m}\left[\frac{\partial^{3}}{\partial y^{3}} p\left(2 y_{m}-y_{0}-y, t\right)\right] Q\left(y_{0}, y_{m}\right) H\left(y_{m}-y_{0}\right) H\left(y_{m}-y\right) .
$$

Analogous to the rearrangement (B2) in the ballistic limit, substituting for the integral on the right-hand side by calculating $\partial^{2} \bar{q} / \partial y^{2}$ from the integral Eq. (20) yields

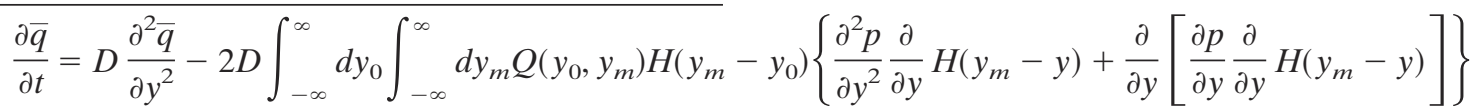

with $p=p\left(2 y_{m}-y_{0}-y, t\right)$ and $\bar{q}=\bar{q}(y, t)$. Substituting for $Q\left(y_{0}, y_{m}\right)$ using the form (B3) and after some straightforward but lengthy manipulations, we obtain the mean evolution Eq. (12) with flux (23) and condensation rate (24).

The identification of the mean flux and mean condensation rate terms can again be verified by direct calculation of the mean condensation rate. Let $d C_{t}$ be the total condensation in the interval $[y, y+d y]$ from time zero to time $t$. The condensation $d C_{t}$ has a contribution $q_{s}(y)-q_{s}(y+d y)$ from all parcels that start at $y_{0}<y^{\prime}$, have maximum excursion $y_{m}>y+d y$, and end at arbitrary $Y(t)=y_{1}$. Since $y_{m} \geq y \geq y^{\prime}$ by the definition (15) of $y^{\prime}$, the condensation $d C_{t}$ is to leading order in $d y$

$$
\begin{aligned}
& d C_{t}= \\
& -\int_{-\infty}^{y^{\prime}} d y_{0} \int_{y}^{\infty} d y_{m} \int_{-\infty}^{\infty} d y_{1} p_{\max }\left(y_{0}, y_{m} \mid y_{1}, t\right) \frac{d}{d y} q_{s}(y) d y .
\end{aligned}
$$

The mean condensation rate is then

$$
\bar{c}(y, t)=\frac{\partial}{\partial t} \frac{d C_{t}}{d y}=-2 D \frac{d q_{s}(y)}{d y} \int_{-\infty}^{y^{\prime}} d y_{0} \int_{y}^{\infty} d y_{m} \int_{-\infty}^{y_{m}} d y_{1} \frac{\partial^{3}}{\partial y_{1}^{3}} p\left(2 y_{m}-y_{1}-y_{0}, t\right)
$$

where we have used the Fokker-Planck Eq. (22) and the maximum excursion PDF (21). Three consecu- tive integrations lead to the mean condensation rate (24). 


\section{REFERENCES}

Bartosch, L., 2001: Generation of colored noise. Int. J. Mod. Phys. C, 12, 851-855.

Boer, G. J., and T. G. Shepherd, 1983: Large-scale twodimensional turbulence in the atmosphere. J. Atmos. Sci., 40, 164-184.

Dessler, A. E., and S. C. Sherwood, 2000: Simulations of tropical upper tropospheric humidity. J. Geophys. Res., 105D, $20155-$ 20163.

Galewsky, J., A. Sobel, and I. Held, 2005: Diagnosis of subtropical humidity dynamics using tracers of last saturation. J. Atmos. Sci., 62, 3353-3367.

Gardiner, C. W., 2004: Handbook of Stochastic Methods. Series in Synergetics, 3d ed. Springer, 415 pp.

Gotoh, T., 1998: Energy spectrum in the inertial and dissipation ranges of two-dimensional steady turbulence. Phys. Rev. E., 57, 2984-2991.

Hagan, P. S., C. R. Doering, and C. D. Levermore, 1989: Mean exit times for particles driven by weakly colored noise. SIAM J. Appl. Math., 49, 1480-1513.

Held, I. M., and B. J. Soden, 2000: Water vapor feedback and global warming. Annu. Rev. Energy Environ., 25, 441-475.

Kelly, K. K., A. F. Tuck, and T. Davies, 1991: Wintertime asymmetry of upper tropospheric water between the northern and southern hemispheres. Nature, 353, 244-247.

Lapeyre, G., and I. M. Held, 2004: The role of moisture in the dynamics and energetics of turbulent baroclinic eddies. J. Atmos. Sci., 61, 1693-1710.

Morse, P. M., and H. Feshbach, 1953: Methods of Theoretical Physics. International Series in Pure and Applied Physics, McGraw-Hill, 1978 pp.

Oetzel, K. G., and G. K. Vallis, 1997: Strain, vortices, and the enstrophy inertial range in two-dimensional turbulence. Phys. Fluids, 9, 2991-3004.

Pasquero, C., A. Provenzale, and A. Babiano, 2001: Parameterization of dispersion in two-dimensional turbulence. J. Fluid Mech., 439, 279-303.

Pierrehumbert, R. T., 1998: Lateral mixing as a source of subtropical water vapor. Geophys. Res. Lett., 25, 151-154.

- and R. Roca, 1998: Evidence for control of Atlantic subtropical humidity by large scale advection. Geophys. Res. Lett., 25, 4537-4540.

-, H. Brogniez, and R. Roca, 2006: On the relative humidity of the atmosphere. The Global Circulation of the Atmosphere: Phenomena, Theory, Challenges, T. Schneider and A. H. Sobel, Eds., Princeton University Press, in press. [Preprint available online at http://geosci.uchicago.edu/ $\mathrm{rtp} 1 /$.
Salathé, E. P., Jr., and D. L. Hartmann, 1997: A trajectory analysis of tropical upper-tropospheric moisture and convection. $J$. Climate, 10, 2533-2547.

Sawford, B., 2001: Turbulent relative dispersion. Annu. Rev. Fluid Mech., 33, 289-317.

Schneider, T., and C. C. Walker, 2006: Self-organization of atmospheric macroturbulence into critical states of weak nonlinear eddy-eddy interactions. J. Atmos. Sci., 63, 1569-1586.

_ , K. L. Smith, P. A. O'Gorman, and C. C. Walker, 2006: A climatology of tropospheric zonal-mean water vapor fields and fluxes in isentropic coordinates. J. Climate, 19, 59175932.

Shaw, N., 1930: The Physical Processes of Weather. Vol. 3, Manual of Meteorology, Cambridge University Press, $445 \mathrm{pp}$

Sherwood, S. C., 1996: Maintenance of the free-tropospheric tropical water vapor distribution. Part II: Simulation by largescale advection. J. Climate, 9, 2919-2934.

Smith, K. S., G. Boccaletti, C. C. Henning, I. Marinov, C. Y. Tam, I. M. Held, and G. K. Vallis, 2002: Turbulent diffusion in the geostrophic inverse cascade. J. Fluid Mech., 469, 13-48.

Soden, B. J., D. L. Jackson, V. Ramaswamy, M. D. Schwarzkopf, and $\mathrm{X}$. Huang, 2005: The radiative signature of upper tropospheric moistening. Science, 310, 841-844.

Stone, P. H., and M. S. Yao, 1990: Development of a twodimensional zonally averaged statistical-dynamical model. Part III: The parameterization of the eddy fluxes of heat and moisture. J. Climate, 3, 726-740.

Thomson, D. J., 1987: Criteria for the selection of stochastic models of particle trajectories in turbulent flows. J. Fluid Mech., 180, 529-556.

, 1990: A stochastic model for the motion of particle pairs in isotropic high-Reynolds-number turbulence, and its application to the problem of concentration variance. J. Fluid Mech., 210, $113-153$.

Uhlenbeck, G. E., and L. S. Ornstein, 1930: On the theory of the Brownian motion. Phys. Rev., 36, 823-841.

Vallis, G. K., 1982: A statistical-dynamical climate model with a simple hydrology cycle. Tellus, 34, 211-227.

van Dop, H., F. T. M. Nieuwstadt, and J. C. R. Hunt, 1985: Random walk models for particle displacements in inhomogeneous unsteady turbulent flows. Phys. Fluids, 28, 1639-1653.

Watterson, I. G., 1998: An analysis of the global water cycle of present and doubled $\mathrm{CO} 2$ climates simulated by the CSIRO general circulation model. J. Geophys. Res., 103, 23113 23129 .

Yang, H., and R. T. Pierrehumbert, 1994: Production of dry air by isentropic mixing. J. Atmos. Sci., 51, 3437-3454. 